\title{
CORRELATION BETWEEN USG AND FNAC IN THYROID SWELLING
}

\author{
Dileep C. $N^{1}$, Yashwanth C. $N^{2}$
}

${ }^{1}$ Resident, Department of Surgery and Emergency Medicine, Shyamanur Shivashankarappa Institute of Medical Sciences, Davanagere, Karnataka.

${ }_{2}^{2}$ Assistant Professor, Department of Surgery and Emergency Medicine, Kerala Medical College, Mangode, Palakkad, Kerala.

\section{ABSTRACT}

\section{BACKGROUND}

Nodular disease of the thyroid is very common, while cancer is less common. A definite diagnosis of either is difficult to make. The general prevalence of thyroid nodules is very high. They are detectable in $5 \%$ of the normal population on clinical examination, in over $48 \%$ on high-resolution ultrasound, and in over $50 \%$ at autopsy.

Aims and Objectives- The purpose of the study was to evaluate clinically the thyroid swelling by sonography and FNAC in various disorders of thyroid gland with histopathological correlation.

\section{MATERIALS AND METHODS}

The present descriptive diagnostic study included 100 patients with clinically palpable thyroid swelling. The patients included in the study were subjected to gray scale and colour Doppler examination of the thyroid gland and fine needle aspiration cytology and histopathological examination.

\section{RESULTS}

Among the 100 patients examined, 30 patients had malignant lesions and 70 patients had benign lesions on final histopathology. All the diagnosis made by ultrasound was compared with FNAC reports. The characteristics of malignant lesions on ultrasonography included single ill-defined microcalcifications, lymphadenopathy and solid predominantly hypoechoic nodule.

\section{CONCLUSION}

Ultrasonography is valuable for identifying many malignant or potentially malignant thyroid nodules. Although, there is some overlap between the ultrasonography appearance of benign nodules and that of malignant nodules, certain ultrasonography features are helpful in differentiating between the two. FNAC can itself diagnose benign conditions and can also be helpful as an aid to ultrasonography features to suggest malignancy, even though it can report malignancy of follicular variety.

\section{KEYWORDS}

High-Resolution Ultrasonography (HRUSG), Fine Needle Aspiration Cytology (FNAC), Thyroid Nodules, Benign, Malignant.

HOW TO CITE THIS ARTICLE: Dileep CN, Yashwanth CN. Correlation between USG and FNAC in Thyroid Swelling. J. Evolution Med. Dent. Sci. 2018;7(16):2011-2013, DOI: 10.14260/jemds/2018/452

\section{BACKGROUND}

Disorders of thyroid gland are amongst the most common endocrine and surgical problems encountered in clinical practice. The prevalence of thyroid nodules is about $3 \%-8 \%$ in the general population and is greater than $50 \%$ after age 65 years.[1,2] The profile of thyroid disorders encountered in paediatric and adolescent age groups in India is similar to that seen in most parts of the world except for the prevalence of iodine deficiency disorders in certain endemic regions of this country. Clinical presentation is most commonly for hypothyroidism, goitres and infrequently for hyperthyroidism. ${ }^{[3]}$ Clinical surveys have shown that $5 \%$ $10 \%$ of the general population have thyroid pathologies including nodular lesions in $2.5 \%-3 \%$ of cases. ${ }^{[4]}$ The prevalence of hyperthyroidism is $2 \%$ in females and $0.6 \%$ in males; hypothyroidism in $4.8 \%$ and $0.9 \%$ and goitre in $2.9 \%$ and $0.4 \%$ respectively. In both sexes, the prevalence increased with age.[5]

'Financial or Other Competing Interest': None.

Submission 03-03-2018, Peer Review 30-03-2018,

Acceptance 04-04-2018, Published 16-04-2018.

Corresponding Author:

Yashwanth C. N,

\#788/3, Saraswathi Nagar

Davanagere-577004. Karnataka.

E-mail:dryashwanthcn@yahoo.co.in

DOI: $10.14260 /$ jemds $/ 2018 / 452$
Thyroid sonography was first introduced in 1966 1967.[6] It has been widely practiced since the 1970 and is now one of the most popular radiological methods of diagnosing thyroid disease.[7] Sonography is commonly the first imaging modality after clinical examination. On the basis of the sonographic findings, selection of additional imaging modalities including CT and MRI imaging can be applied more judiciously. Fine needle aspiration cytology (FNAC) is now a well-established, first line, simple and quick screening test as well as the diagnostic tool for surgical and non-surgical goitres. Limitation of FNAC is mainly because of inadequate sampling in experience of the pathologist and overlapping cytological features.[8] Ultrasonography is an easily accessible, non-invasive way to image the thyroid gland and its pathology. It helps to pinpoint a possible thyroid abnormality at an early stage and includes the elements of differential diagnosis that result in subsequent thorough examination and timely treatment in appropriate cases. In addition to facilitating the diagnosis of clinically apparent nodules, the widespread use of ultrasonography has resulted in uncovering a multitude of clinically unapparent thyroid nodules, while differentiating majority of them which were benign from malignant nodules. The present study was aimed to determine the role of high resolution ultrasonography in the evaluation of lesions of the thyroid with FNAC correlation and compared with other studies. 


\section{MATERIALS AND METHODS}

The present descriptive diagnostic study was conducted in the Department of General Surgery and Department of Radiology in Kerala Medical College. This study includes a total of 100 patients (89 female and 11 male) of all age groups admitted in surgical wards during the period. The patients included in the study were subjected to gray scale and colour Doppler examination of the thyroid gland and fine needle aspiration cytology and histopathological examination. FNAC is considered the gold standard diagnostic test in the evaluation of a thyroid nodule. By using this we accessed the specificity, sensitivity, PPV and NPV of the FNAC in detecting thyroid swelling.

\section{Inclusion Criteria}

All the patients coming with lump in the anterior aspect of neck were included in the study.

\section{Exclusion Criteria}

- Previously diagnosed cases of benign disease and thyroid malignancies.

- Cases undergoing treatment.

- Diagnosed cases of carcinoma thyroid on follow-up for residual disease or recurrence.

\section{Examination Method}

A brief history was taken, and physical examination was carried out. Patients were subjected to high-resolution ultrasonographic (HRUSG) examination and the suspected lesions are further subjected to fine needle aspiration cytology following informed verbal consent. The acoustic power in the Doppler mode was limited to that recommended by the current US Food and Drug Administration guidelines for thyroid scanning.

\section{Technique of USG Neck}

The thyroid gland was located superficially, and hence high resolution real time ultrasonography can demonstrate normal thyroid anatomy and pathological conditions with remarkable clarity. High frequency transducers (7.5 - 15.0 MHZ) currently provide both deep ultrasound penetration upto $5 \mathrm{~cm}$ and high definition images with a resolution of 0.7 - $1.0 \mathrm{~mm}$. Linear array transducers are preferred to sector transducers, because of wider near field of view.

\section{Technique of FNAC of Thyroid Lesion}

The swelling was uncured with $23-\mathrm{G}$ needle attached to a 10 cc disposable syringe after cleaning and draping the patient. The material was aspirated from the swelling and two smears are made. First slide was air dried and second was fixed wet in ether. Air dried smear was stained with MGG stain, while ether fixed smear was stained with $\mathrm{H}$ and $\mathrm{E}$.

\section{Statistical Methods}

Fisher's exact test was used and also sensitivity, specificity, positive predictive value (PPV) and negative predictive value (NPV) were calculated from the data tabulated correlating sonographic and FNAC characteristics.

\section{RESULTS}

In the present study, out of 100 patients 89 were female and 11 were male. Out of 100 lesions, 68 (64 were benign and 4 were malignant) were hyperechoic, 27 ( 9 were benign and 18 were malignant) were hypoechoic and 3 were isoechoic and 2 were anechoic. If hypoechoic nature of the lesion is considered as an independent factor in identifying malignancy in a thyroid lesion it could detect malignancy with a sensitivity, specificity, positive predictive value, negative predictive value of $72 \%, 88 \%, 67 \%$ and $90 \%$ respectively. Out of 100 lesions seen at HRUSG 70 lesions (65 benign, 5 malignant) were well-defined, out of which as diagnosed by FNAC 30 lesions (20 malignant, 10 benign) were ill-defined, of which if ill-defined borders of the lesion were considered as an independent factor indicative of malignancy it could detect a malignant lesion with sensitivity, specificity, positive and negative predictive value of $80 \%$, $87 \%, 67 \%$ and $93 \%$ respectively. Out of 100 lesions seen at HRUSG, 70 lesions (65 benign, 5 malignant) showed macrocalcifications, 27 lesions (17 malignant, 10 benign) showed microcalcifications. If microcalcification was considered as an independent factor identifying malignant lesion, it could detect malignancy with sensitivity, specificity positive predictive value and negative predictive value of $68 \%, 87 \%, 63 \%$ and $89 \%$ respectively. Out of 100 lesions seen at HRUSG, 67 lesions ( 63 benign, 4 turned out to be malignant) showed peripheral vascularity and 28 lesions (12 benign, 16 malignant) showed central vascularity. If central vascularity was considered as an independent factor for malignancy it would detect malignancy with a sensitivity, specificity, positive predictive value and negative predictive value of $64 \%, 84 \%, 57 \%$ and $88 \%$ respectively. Out of 100 lesions seen at HRUSG, 66 lesions (62 begin, 4 turned out to be malignant) showed $\mathrm{A} / \mathrm{T}$ ratio less than one, 34 lesions (13 benign 21 malignant) showed $\mathrm{A} / \mathrm{T}$ ratio more than one. If $\mathrm{A} / \mathrm{T}$ ratio more than one was considered as an independent factor for malignancy it would detect malignancy with a sensitivity, specificity, positive predictive value and negative predictive value of $84 \%, 83 \%, 62 \%$ and $94 \%$ respectively. Out of 100 cases seen at HRUSG number of cases identified as benign in USG was 70, out of which 61 were benign and 9 turned out to be malignant in FNAC. Number of cases identified as malignant in USG was 30 , out of which 16 were malignant and 14 turned out be benign in FNAC. The overall sensitivity, specificity, positive value and negative predictive value of ultrasonography in identifying a malignant lesion were $64 \%$ and $87 \%$ respectively. Hence, USG is good at ruling out a malignancy lesion, thus playing a role in management.

\begin{tabular}{|c|c|c|c|c|}
\hline Variables & $\begin{array}{c}\text { M.C. } \\
\text { Frateset, } \\
\text { et al[12] }\end{array}$ & $\begin{array}{l}\text { Enrido } \\
\text { Papini, } \\
\text { et al[10] }\end{array}$ & $\begin{array}{c}\text { Won-Jin } \\
\text { Moon, } \\
\text { et al[11] }\end{array}$ & $\begin{array}{c}\text { Present } \\
\text { Study }\end{array}$ \\
\hline $\begin{array}{l}\text { Hypoechoic } \\
\text { nodule }\end{array}$ & $26.5-87.1$ & $87 \%$ & $87 \%$ & $72 \%$ \\
\hline Irregular margins & $17.4-77.5$ & $77 \%$ & $48 \%$ & $80 \%$ \\
\hline $\begin{array}{c}\text { Central } \\
\text { vascularity }\end{array}$ & $54.3-74.2$ & $75 \%$ & - & $64 \%$ \\
\hline Microcalcifications & $\begin{array}{c}26.1- \\
59.1 \%\end{array}$ & $29 \%$ & $44 \%$ & $68 \%$ \\
\hline $\begin{array}{l}\text { A/T more than } \\
\text { one }\end{array}$ & 32.7 & - & $40 \%$ & $84 \%$ \\
\hline \multicolumn{5}{|c|}{ Table 1} \\
\hline
\end{tabular}




\section{DISCUSSION}

High-resolution ultrasound has become the first line imaging modality for evaluation of the thyroid gland due to excellent visualisation of the thyroid parenchyma. It is highly sensitive in detecting small nodules, calcification, septations and cysts as well as in guiding fine needle aspiration biopsies. Thyroid nodules are very common and may be observed on ultrasonography (US) in $50 \%$ of the adult population.[9] The most common cause of benign thyroid nodules is nodular hyperplasia, although less than $7 \%$ of thyroid nodules are malignant.[10] The present study was done to study the role of high-resolution ultrasonography in the evaluation of thyroid lesions in correlation with FNAC of the same lesions. The present study when compared with other studies in the literature showed similar trends in identifying a thyroid malignancy. The individual variables considered by several authors were same as those considered in the present study and they showed variable correlations with the sensitivities. The sensitivity rate for malignancy on HRUSG in the study by Won-Jin Moon et al were as follows: Hypoechoic in nature (87\%), ill-defined margins (48\%), microcalcifications (44\%) and $\mathrm{A} / \mathrm{T}$ ratio more than one $(40 \%)$. The presence of microcalcifications was the least sensitive variable in predicting malignancy in the above three studies, while sensitivity was significantly lower in the studies by both Mary C Frates et al and Enrido Papini et al compared to our study. The present study on comparison with the study made by Mary C Frateset et al showed highest correlation with hypoechogenicity of the nodule and with central vascularity. It also showed lowest concentration with the sonographic features A/T ratio more than one, while irregular margins and central vascularity showed variable correlation. In the study by Enrido Papini et al sensitivity rates for malignancy on HRUSG were as follows: In decreasing order hypoechoic nature $(87 \%)$, ill-defined margins $(77 \%)$, central vascularity (75\%) and microcalcifications (29\%).[10] In present study, irregular margins and central vascularity showed highest correlation. The study by Enrido Papini et al has not considered $\mathrm{A} / \mathrm{T}$ ratio more than one, which showed the highest sensitivity to predict malignancy in our study, while hypoechogenicity of nodule was more sensitive. Several US features have been found to be associated with an increased risk of thyroid cancer including presence of calcifications, hypoechogenicity, irregular margins, predominantly solid composition and intranodular vascularity; however, the sensitivities, specificities and negative and positive predictive values for these criteria are extremely variable from study to study and no US feature has both high sensitivity and high positive predictive value for thyroid cancer while the combination of factors improves the positive predictive value of US to some extent in diagnosing a malignant lesion.

\section{CONCLUSION}

HRUSG is extremely useful in showing whether the palpable mass is within thyroid or adjacent to the thyroid. Highest incidence of thyroid disease was in the population age group of 20 - 50 years. Majority of patients were female and of the 100 patients 70 lesions were benign and 30 lesions were malignant. The sensitivity of the lesion on sonography correlated with malignancy on FNAC as follows: Hypoechoic $72 \%$, ill-defined border $80 \%$, microcalcification $68 \%$, central vascularity $64 \%$ and $\mathrm{A} / \mathrm{T}$ ratio $84 \%$. From this study out of 100 cases seen at HRUSG followed by FNAC correlation, the overall sensitivity, specificity, positive predictive value and negative predictive value of ultrasonography in identifying a malignant lesion was $64 \%, 81 \%, 53 \%$ and $87 \%$ respectively. This study showed that HRUSG due to its excellent resolution is very sensitive in detecting the confines of lesion to the thyroid and local invasion into nearby structures. HRUSG is also highly significant in detecting cervical lymph nodes associated with thyroid lesions, characterising them as benign or malignant. Hence, USG is good at ruling out a malignant lesion, thus playing a role in management.

\section{REFERENCES}

[1] Wiest PW, Hartshorne MF, Inskip PD, et al. Thyroid palpation versus high-resolution thyroid ultrasonography in the detection of nodules. J Ultrasound Med 1998;17(8):487-96.

[2] Morstensen JD, Woolner LB, Bennett WA. Gross and microscopic findings in clinically normal thyroid glands. J Clin Endocrinal Metab 1955;15(10):1270-80.

[3] Desai MP. Disorders of thyroid gland in India. Indian J Pediatr 1997;64(1):11-20.

[4] Kharchenko VP, Kotlyarov PM, Mogutov MS, et al. Ultrasound diagnostic of thyroid diseases. Springer Verlag Berlin Heidelberg 2010.

[5] Bjoro T, Holmen J, Kruger 0, et al. Prevalence of thyroid disease, thyroid dysfunction and thyroid peroxidase antibodies in a large, unselected population. The health study of Nord - Trondelag (HUNT). European Journal of Endocrinology 2000;143(5):639-47.

[6] Fujimoto F, Oka A, Omoto R, et al. Ultrasound scanning of the thyroid gland as a new diagnostic approach. Ultrasonics 1967;5(3):177-80.

[7] Policeni BA, Smoker WR, Reede DL. Anatomy and embryology of the thyroid and parathyroid glands. Semin Ultrasound CT MRI 2012;33(2):104-14.

[8] Khafagi F, Wright G, Castles H, et al. Screening for thyroid malignancy: the role of fine needle aspiration biopsy. Med J Aust 1988;149(6):302-3, 306-7.

[9] Frates MC, Benson CB, Charboneau JW, et al. Management of thyroid nodules detected at US: Society of radiologists in Ultrasound consensus conference statement. Radiology 2005;237(3):794800.

[10] Papini E, Guglielmi R, Bianchini A, et al. Risk of malignancy in non-palpable thyroid nodules: predictive value of ultrasound and color - Doppler features. J Clin Endocrinol Metab 2002;87(5):1941-6. 